\title{
Materiales carbonosos obtenidos a partir del reciclado de PET
}

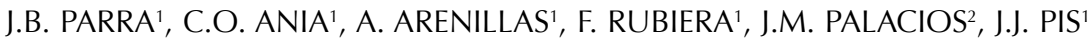 \\ ${ }^{1}$ Instituto Nacional del Carbón. Campus U.A.M., C.S.I.C., 33080 Oviedo \\ 2 Instituto de Catálisis y Petroleoquímica, C.S.I.C. Cantoblanco, 28049 Madrid
}

\begin{abstract}
El polietilentereftalato o tereftalato de polietileno, PET, es un poliéster termoplástico ampliamente utilizado. Debido a su gran producción y consumo se genera un gran volumen de PET usado que es necesario tratar. En la actualidad, la única alternativa utilizada para la minimización de residuos de PET de pureza media/baja es la incineración. Por lo tanto, se hace necesaria la búsqueda de otras opciones que aprovechen en mayor medida el potencial de este residuo y que, a la vez, sean rentables. En este trabajo se muestra cómo el PET proveniente de botellas usadas puede ser una fuente de materiales carbonosos que desarrollan una microporosidad controlada, por lo que su campo de aplicación puede ser muy amplio.

Palabras clave: PET, reciclado, carbones activos, textura

\section{Carbonaceous materials from recycled Pet}

Poly(ethyleneterephthalate), PET, is nowadays one of the polymers more widely used. However, due to its big production, it accounts for a large part of the wastes generated and it becomes necessary to minimise them. The PET incineration with energy recovery is the most common way to eliminate these residues. Further research is needed in order to find alternative processes to recycling PET. In this work, it is shown that the post-consumer PET is an interesting source of carbonaceous materials that develop a well controlled microporosity, giving the possibility of application in several fields.
\end{abstract}

Key words: PET, recycling, active carbons, textural properties

\section{INTRODUCCIÓN}

En el año 2001, la producción de PET en Europa fue superior a $300 \mathrm{kt}$, un $20 \%$ más que en el año 2000 (1). Las predicciones indican un aumento progresivo a lo largo de los próximos años, para alcanzar 700 kt en el año 2006. La principal aplicación del PET es la fabricación de fibras, aunque también es de destacar la fabricación de botellas y moldes.

Este polímero se utiliza ampliamente en la industria alimentaria debido a su inercia frente a los alimentos, su transparencia, la dificultad para ser agujereado, su despreciable permeabilidad frente al $\mathrm{CO}_{2}$ y su maleabilidad.

Como consecuencia de su gran producción y consumo se genera un gran volumen de PET usado que es necesario tratar. Las distintas alternativas para su reutilización dependen del grado de pureza del polímero; cuando la pureza es alta se puede volver a fundir y reprocesar para obtener nuevamente PET, en otros casos se recurre a su incineración aprovechándose como fuente de energía. Sin embargo, el reciclado de PET para la obtención de otros compuestos mediante despolimerización (vg. ácido tereftálico y etilenglicol), es aún inviable debido a la complejidad y a los altos costes del proceso (1-2). Esto impone la búsqueda de nuevas alternativas a los residuos de PET.

Recientemente se han publicado diversos trabajos que estudian las propiedades de compuestos obtenidos a partir del reciclado de PET, así como el comportamiento de mezclas de PET con diversas sustancias (2-6). En ambos casos, el objetivo es obtener productos de mayor valor añadido que hagan el proceso rentable.

László y Szücs (7) propusieron la obtención de carbón activo a partir de PET granulado virgen, utilizando vapor de agua como agente activante. En el presente trabajo se ha estudiado la posibilidad de reutilizar el PET usado para la obtención de un material carbonoso con superficies específicas muy variables (entre 300 y $2500 \mathrm{~m}^{2} \mathrm{~g}^{-1}$ ). De esta forma se obtiene un material de alto valor añadido, a través de un proceso relativamente sencillo y reciclando residuos de PET.

\section{PARTE EXPERIMENTAL}

PET usado procedente de botellas, previamente cortado, se sometió a un proceso de pirólisis en un horno vertical bajo atmósfera de nitrógeno, calentando hasta una temperatura de $725^{\circ} \mathrm{C}$. Se obtuvo un residuo negro esponjoso de aspecto vítreo, con un rendimiento del $22 \%$. Dicho residuo se molió a un tamaño inferior a $1 \mathrm{~mm}$, seleccionándose para el presente estudio la fracción granulométrica entre 0,5 y $1 \mathrm{~mm}$, que se ha denominado PM.

Con objeto de optimizar la temperatura de activación del material pirolizado, se realizó un estudio previo en termobalanza para evaluar la reactividad de dicho material frente al $\mathrm{CO}_{2}$. El estudio puso de manifiesto la baja reactividad del material, que no comienza a reaccionar con $\mathrm{CO}_{2}$ hasta aproximadamente $925^{\circ} \mathrm{C}$. De acuerdo con estos resultados, se seleccionó dicha temperatura para la etapa de activación, utilizándose un flujo de $\mathrm{CO}_{2}$ de $10 \mathrm{~cm}^{3} \mathrm{~min}^{-1}$. Se obtuvieron así una serie de materiales con distinto grado de activación $(0,12,35,58$ y $76 \%)$ que se denominaron PM0, PM12, PM35, PM58 y PM76, respectivamente.

La caracterización de las muestras se realizó mediante isotermas de adsorción de $\mathrm{N}_{2}$ a $77 \mathrm{~K}$ en un equipo ASAP 2010 de Micromeritics. Los difractogramas de rayos $X$ se obtuvieron en un equipo Siemens D5000, utilizando radiación CuK $(\square=0,15406 \mathrm{~nm}), 0,015^{\circ}$ de tamaño de paso y $3 \mathrm{~s}$ de tiempo de paso. La textura óptica de las muestras se llevó a cabo mediante microscopia electrónica de barrido, utilizando un equipo Zeiss modelo DSM 942. 


\section{RESULTADOS Y DISCUSIÓN}

El análisis elemental de las muestras estudiadas se muestra en la Tabla 1. Estos materiales se caracterizan por la ausencia total de materia mineral y un alto contenido en carbono (superior al 98,2\% en peso).

TABla 1. CARACTERIZACIÓN DE LAS MUESTRAS ESTUDIADAS

\begin{tabular}{|l|c|c|c|c|c|c|c|}
\hline \multirow{2}{*}{ Muestra } & \multicolumn{3}{|c|}{$\begin{array}{c}\text { Análisis elemental } \\
\text { (\% masa) }\end{array}$} & $\begin{array}{c}\text { Adsorción } \\
\text { nitrógeno }\end{array}$ & \multicolumn{3}{c|}{$\begin{array}{c}\text { Difracción } \\
\text { de rayos X (nm) }\end{array}$} \\
\cline { 2 - 8 } & $\mathrm{C}$ & $\mathrm{O}$ & $\mathrm{H}$ & $\mathrm{S}_{\mathrm{BET}}\left(\mathrm{m}^{2} \mathrm{~g}^{-1}\right)$ & $\mathrm{d}(002)$ & $\mathrm{Lc}$ & $\mathrm{La}$ \\
\hline PM & 96,6 & 1,2 & 1,8 & - & 0,38 & 0,99 & 3,29 \\
\hline PM0 & 98,2 & 0,9 & 0,5 & 340 & 0,38 & 1,05 & 3,52 \\
\hline PM12 & 98,8 & 0,6 & 0,3 & 668 & 0,38 & 1,07 & 3,69 \\
\hline PM35 & 98,9 & 0,6 & 0,3 & 1405 & 0,38 & 1,12 & 3,98 \\
\hline PM58 & 98,7 & 0,6 & 0,2 & 1920 & 0,39 & 1,17 & 4,48 \\
\hline PM76 & 99,0 & 0,5 & 0,2 & 2468 & 0,44 & 1,37 & 5,03 \\
\hline
\end{tabular}

\subsection{Microscopía electrónica de barrido}

Todos los materiales obtenidos en el presente trabajo presentan un aspecto vítreo. Su observación mediante microscopía electrónica de barrido revela la presencia de superficies lisas con ruptura concoidea. La muestra sin activar (PM0) no presenta porosidad, tal y como se observa en la Figura 1a. A medida que avanza el proceso de activación se aprecia el desarrollo de porosidad en la muestra. La Figura 1b corresponde a la muestra mas activada (PM76), observándose claramente la presencia de gran número de poros con un tamaño aproximado de 0,1 $\mu \mathrm{m}$. En el caso de materiales activados a partir de carbón mineral es normal la presencia de grietas o fisuras a lo largo de su superficie. Sin embargo en las muestras activadas a partir de PET hay que destacar la total ausencia de estas grietas, por lo que las partículas no se rompen y su tamaño no cambia durante el proceso de activación.

\subsection{Difracción de rayos $X$}

En la Tabla 1 se presentan también distintos parámetros estructurales derivados de los análisis de difracción de rayos X: el espaciado entre capas, $\mathrm{d}_{002^{\prime}} \mathrm{y}$ los tamaños cristalinos, La y Lc. Se observa claramente un aumento de los parámetros La y Lc con el grado de activación. De forma general puede deducirse que al incrementarse el grado de activación se aumenta el tamaño de los microcristales de la estructura turbostrátrica, sobre todo en la dimensión La, que pasa de 3,29 nm en la muestra PM a 5,03 nm para PM76. Sin embargo, el valor de Lc sólo aumenta de 0,99 nm para PM hasta 1,37 nm para la muestra más activada. De estos resultados parece inferirse que la activación transcurre por reacción de las estructuras más desordenadas en la dirección de las capas grafíticas.

\subsection{Caracterización textural}

En la Tabla 1 se muestran los valores de superficie específica, calculados mediante la ecuación de BET a partir de las isotermas de adsorción de nitrógeno a $77 \mathrm{~K}$, para las diferentes muestras estudiadas. La superficie específica presenta un gran aumento con la activación, pasando de $340 \mathrm{~m}^{2} \mathrm{~g}^{-1}$ para la muestra sin activar (PM0) a $2468 \mathrm{~m}^{2} \mathrm{~g}^{-1}$ en el caso de la muestra con un grado de quemado del 76\% (PM76). En todas las muestras la mayor parte del volumen adsorbido de nitrógeno se debe a la microporosidad presente en las mismas (8). El estudio de
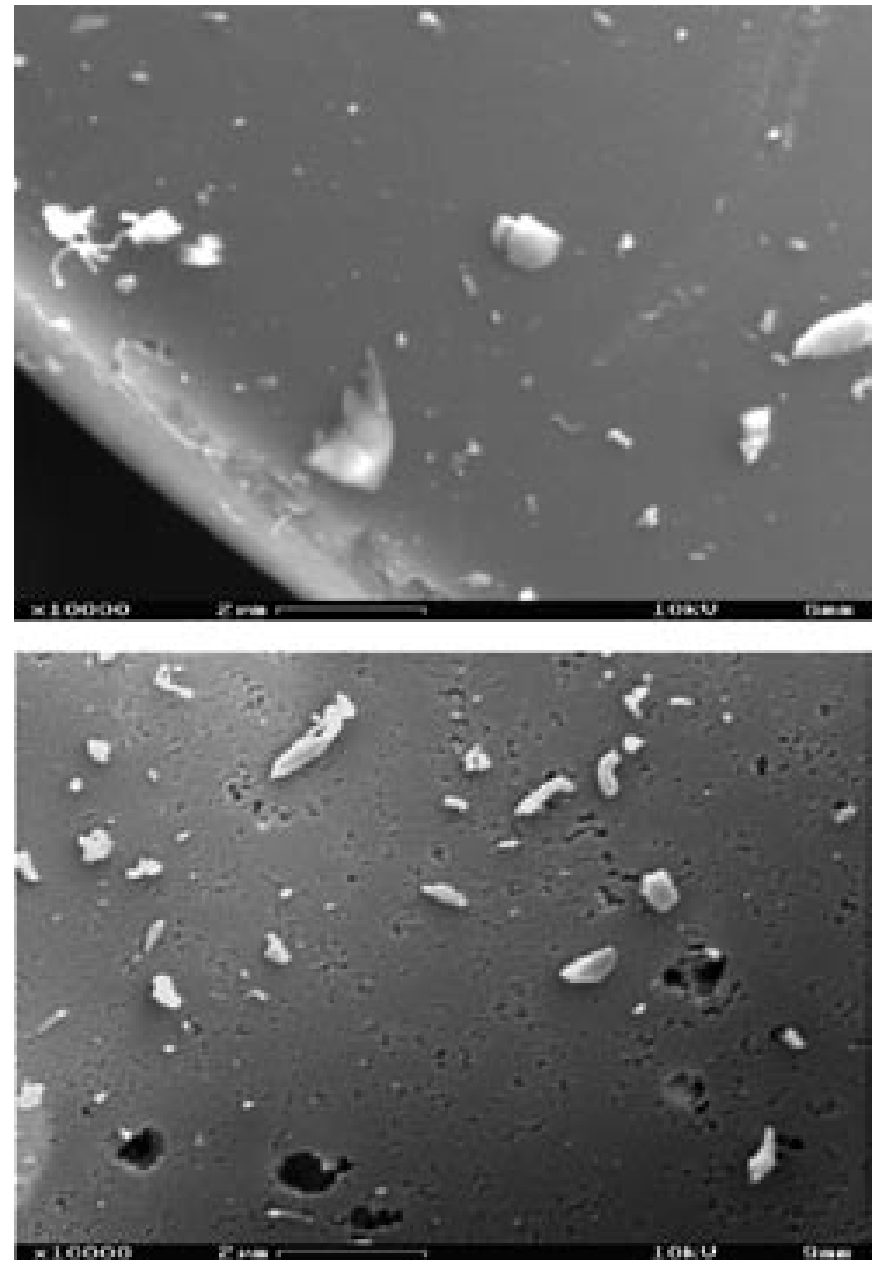

Figura 1.Imágenes obtenidas mediante microscopía electrónica de barrido de las muestras PM0 (a) y PM76 (b).

la evolución de la microporosidad con el grado de activación, se ha llevado a cabo mediante la aplicación del método de Horváth-Kawazoe -HK- (9); aunque se debe señalar que existe cierta controversia respecto a los valores absolutos de ancho de poro, L, calculados por este método (10).

En la Figura 2 se presentan las curvas de volumen acumulado y distribución del volumen de microporos obtenidas a partir del método HK para las muestras activadas. La muestra PM0 presenta una adsorción de $\mathrm{N}_{2}$ despreciable a bajas presiones, por lo que no se ha podido aplicar este método.

Comparando las curvas de volúmenes acumulados de poro se observa un claro incremento del volumen total de microporos, desde $0,246 \mathrm{~cm}^{3} \mathrm{~g}^{-1}$ para PM12 hasta $0,781 \mathrm{~cm}^{3} \mathrm{~g}^{-1}$ para PM76. Asimismo, de la pendiente de la curva en la zona de $2 \mathrm{~nm}$ se infiere la presencia de porosidad de mayor tamaño a medida que aumenta el grado de activación, ya que las curvas presentan una pendiente creciente.

Las curvas derivadas del volumen de poros respecto al ancho medio de los mismos permiten visualizar la evolución de la porosidad con el aumento del grado de activación. La curva perteneciente a la muestra PM12 presenta un solo máximo muy estrecho para $\mathrm{L}=0,65$ $\mathrm{nm}$, disminuyendo bruscamente y no presentando adsorción apreciable a valores mayores de unos $0,8 \mathrm{~nm}$. Teniendo en cuenta que la adsorción de $\mathrm{N}_{2}$ de la muestra PM0 es muy inferior a la de la muestra PM12 y que además la isoterma presenta una histéresis total a baja 
presión, parece que la activación al $12 \%$ provoca un aumento del tamaño de los microporos generados durante la pirólisis. A pesar de esto, todos los microporos presentes en PM12 son de pequeño tamaño, ya que la mitad del volumen de microporos presenta anchos de poro menores de $0,7 \mathrm{~nm}$. La activación a un grado de quemado del $35 \%$ duplica el volumen de microporos con respecto al de la muestra activada al 12\%. Además, parece que la adsorción también tiene lugar en los microporos más pequeños, ya que presentan un máximo muy estrecho a bajas presiones (del orden de $10^{-6} \mathrm{p} / \mathrm{p}^{0}$ ). Por otra parte, en la muestra PM35 se empiezan a desarrollar poros de mayor tamaño, debido a la reacción de las paredes de los poros ya creados con el gas activante $\left(\mathrm{CO}_{2}\right)$. La curva de distribución de poros del carbón activado al 58\% presenta cambios significativos en comparación con las muestras anteriores. Como se puede observar en la Figura 2, el máximo se produce a valores inferiores al de la muestra PM12. Al mismo tiempo aparecen dos nuevos picos centrados en valores del ancho de poro de 0,66 y $0,78 \mathrm{~nm}$. Todo indica que estos poros se producen por evolución de los microporos más pequeños presentes en la muestra. Al continuar activando (muestra PM76) se completa esta evolución. La distribución de microporos presenta en esta muestra una distribución casi gaussiana abarcando poros con $\mathrm{L}$ desde $0,6 \mathrm{~nm}$ hasta $1,5 \mathrm{~nm}$, aunque de la forma de la curva acumulada de volúmenes de poro se puede comprobar que existen poros mayores.

\section{CONCLUSIONES}

Los resultados presentados en este trabajo indican que los materiales carbonosos obtenidos a partir de PET usado presentan una evolución de la microporosidad que puede ser controlada y, por lo tanto, el campo de aplicaciones de estos materiales es muy amplio. Se ha comprobado que tienen una gran eficacia de adsorción de fenol, por lo que pueden ser utilizados como adsorbentes en la depuración de efluentes. Por otro lado, presentan buenas perspectivas para almacenamiento de gases, como $\mathrm{H}_{2}$ y/o $\mathrm{CH}_{4}$. Los materiales obtenidos a bajo grado de activación, y que presentan una distribución de microporosidad estrecha, pueden ser utilizados como tamices moleculares. Por otra parte, pueden ser la base para la obtención de catalizadores, previa funcionalización con ácido nítrico, lo cual combinado con las propiedades de un tamiz molecular le confieren aplicaciones específicas interesantes en la depuración de gases.

\section{BIBLIOGRAFÍA}

\section{1. www.petcore.org}

2. M.S. Farahat, D. E. Nikles. "On the UV curability and mechanical properties of novel binder systems derived from poly(ethyleneterephthalate) (PET) waste for solventless magnetic tape manufacturing, $2^{\mathrm{a}}$ Methacrylated Oligoesters» Macromol. Mater. Eng. 287 353-362 (2002).

3. M. Pluta, Z. Bartczak, A. Pawlak, A. Galeski, M. Pracella. «Phase structure and viscoelastic properties of compatibilized blends of PET and HDPE recyclates». J. Appl. Polym. Sci. 82 1423-1436 (2001).

4. M. Pracella, F. Pazzagli, A. Galeski. «Reactive compatibilization and properties of recycled poly(ethyleneterephthalate)/polyethylene blends». Polymer Bull. 48 67-74 (2002).

5. A. Ruvolo-Filho, A. de Fátima Barros. «Correlation between thermal properties and conformational changes in poly(ethyleneterephthalate)/ poly(etherimide) blends». Polymer Degradation and Stability 73 467470 (2001).
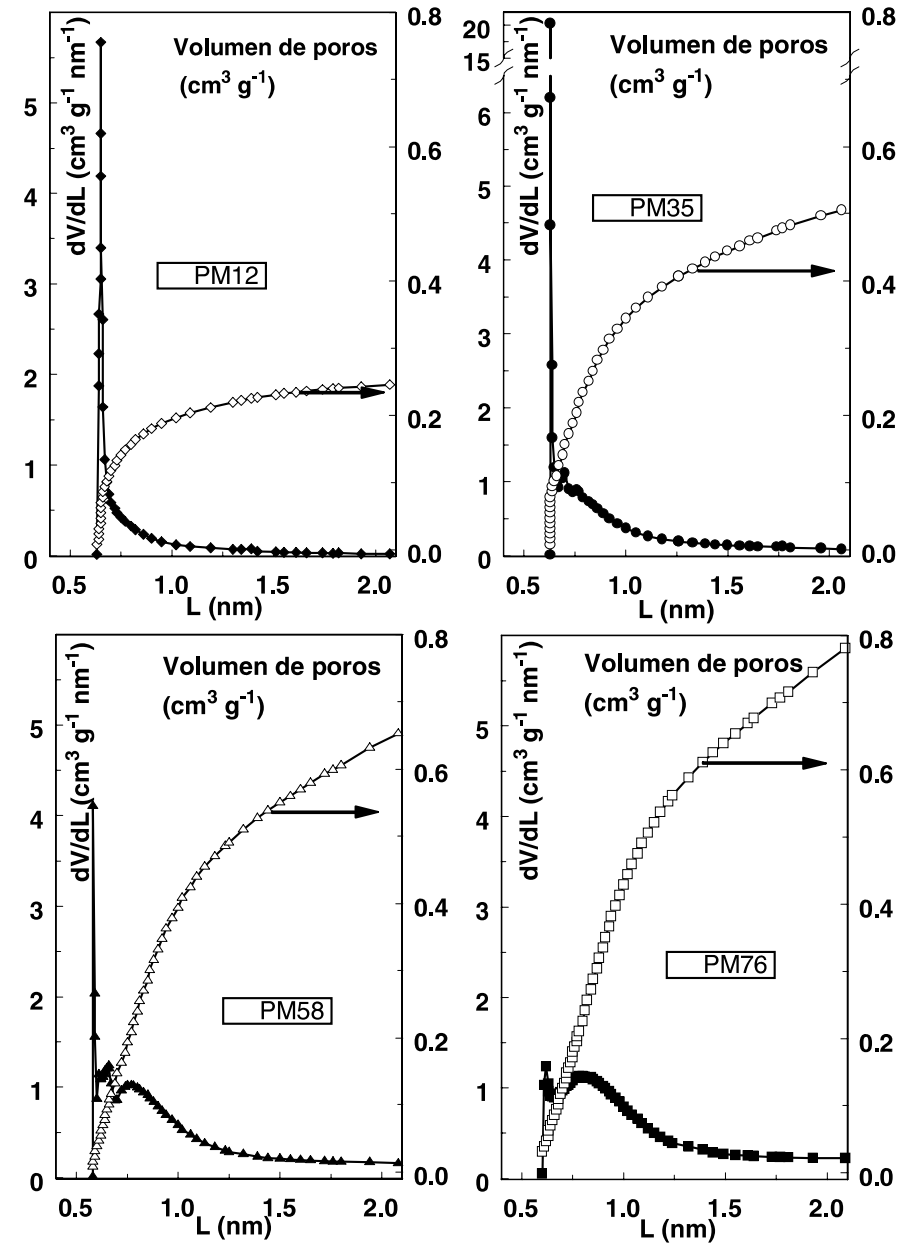

Figura 2. Volumen acumulado y distribución del volumen de microporos en las muestras activadas, obtenidos a partir del método de Horváth-Kawazoe.

6. G. Burillo, P. Herrera-Franco, M. Vazquez, E. Adem. «Compatibilization of recycled and virgin PET with radiation-oxidized HDPE». Radiation Physics and Chemistry 63 241-244 (2002).

7. K. László, A. Szücs «Surface characterization of polyethyleneterephthalate (PET) based activated carbon and the effect of $\mathrm{pH}$ on its adsorption capacity from aqueous phenol and 2,3,4 trichlorophenol solutions». Carbon 39 19451953 (2001).

8. J.B. Parra, C.O. Ania, A. Arenillas, J.M. Palacios, J.J. Pis. «Propiedades texturales de carbones activos obtenidos a partir de residuos de tereftalato de polietileno» pp 89-90 en XXVII Reunión Ibérica de Adsorción" León, septiembre 2002

9. G. Horváth, K. Kawazoe. «Method for the calculation of effective pore size distribution in molecular sieve carbon». J. Chem. Eng. Japan 16 470-475 (1983).

10. F. Rouquerol, J. Rouquerol, K.Sing. «Assessment of microporosity» pp. 232, en "Adsorption by powders \& pororus solids", Academic Press, Londres (UK) 1999.

Recibido: 1.2 .03

Aceptado: 30.11 .03 